\title{
MECHANISMS OF NONUNIFORM LINE BROADENING
}

\author{
Z. Wilamowski, H. Przybylińska \\ Institute of Physics, Polish Academy of Sciences \\ Al. Lotników 32/46, 02-668 Warszawa, Poland \\ AND W. JANTSCH \\ Johannes Kepler Universität, 4040 Linz-Auhof, Austria
}

\begin{abstract}
When the spin relaxation time of paramagnetic impurities at different magnetic sites differs by orders of magnitude then instead of resonance line broadening, rather a decay of the line amplitude is expected. In particular, the $s p-d$ coupling of magnetic impurities to shallow impurities leads to a nonuniform distribution of the longitudinal relaxation rates of local spins.
\end{abstract}

PACS numbers: 76.20.+q, 76.30.-v

According to the general theory of magnetic resonance the integrated intensity of the resonance line depends on the total number of spins involved in this resonance and on temperature, where the temperature dependence of the line intensity should reflect the temperature changes of the static magnetic susceptibility. A decay of the resonance amplitude is thus usually caused by line broadening. In some cases, however, the line decays without a visible broadening and the temperature dependence of the line amplitude does not correlate with the temperature changes of the susceptibility $[1,2]$. Such a behavior can originate from a strongly nonuniform distribution of the relaxation times responsible for line broadening. When the relaxation rates of localized spins, because of the variety of their local configurations, differ by orders of magnitude, then the resonances occurring at centers which relax very fast are so broad that they do not contribute to the observed line amplitude. In this paper we analyze the decay of the spectral line. We show that for a strongly nonuniform distribution of relaxation rates the line intensity, scaled as a product of the line amplitude and the square of the peak-peak linewidth, is determined by the integral over the rate distribution, beginning from the lowest rates and ending with the value corresponding to a native relaxation rate which originates from another relaxation mechanism.

We analyze also the consequences of a nonuniform distribution of the longitudinal relaxation mechanisms, showing that mechanisms of such a type manifest themselves by a characteristic dependence of the line amplitude on the microwave 
power. For a given power some fraction of the spins is completely saturated and the corresponding spectrum is totally quenched, while other spins still contribute to the spectrum. As a consequence, we observe an extended plateau in the dependence of the line amplitude vs. microwave power. The width of the plateau corresponds to the dispersion of the relaxation times.

The effect of nonuniform spin relaxation can be observed in semiconductors if the lifetimes of impurities in a given valence state are spread due to fluctuations in the ionization energy [1], or in metals if the relaxation proceeds mainly via RKKY coupling to other local magnetic moments [2]. Such a probability distribution of longitudinal relaxation rates is expected in mixed valence $\mathrm{HgSe}: \mathrm{Fe}$ [1], where the dominant relaxation mechanism is proportional to the square of the indirect RKKY coupling between $\mathrm{Fe}^{2+}$ and $\mathrm{Fe}^{3+}$ centers. Since it is not uniform, the EPR spectrum of the $\mathrm{Fe}^{3+}$ ions decays without appreciable broadening both with increasing temperature and $\mathrm{Fe}$ concentration. In this paper we analyze a new spin relaxation mechanism. The $s p-d$ coupling between the spins of the magnetic $d$-ion and shallow $s p$-impurities seems to be the dominant mechanism of longitudinal relaxation in diluted magnetic semiconductors. We show that this mechanism can be strongly nonuniform, and that an evaluation of the nonuniformity allows one to estimate the electrical properties of the semiconductor. In particular, the metal insulator transition can be investigated.

We assume that the longitudinal relaxation rate of the $d$-spin, which is caused by a time fluctuation of the shallow $s p$-impurity state, is proportional to the square of the $s p-d$ exchange coupling, $J_{s p-d}^{2}$, and that the coupling constant is proportional to the square of the shallow impurity wave function. Summarizing, the relaxation rate of the $d$-spin to a single $s p$-spin is characterized by an exponential dependence on distance, $J_{s p-d}^{2}(r)=J_{0}^{2} \exp \left(-4 r / r_{\mathrm{B}}\right)$.

We consider a system of shallow impurities distributed at random positions, $r_{j}$, to which the relaxation may occur, and sum over all the contributions from every such center to a random position of the $d$-spin $R$. We assume that the sum of the $J_{s p-d}^{2}\left(\boldsymbol{R}-\boldsymbol{r}_{j}\right)$ is proportional to the relaxation rate, $\nu(\boldsymbol{R})$, and calculate the probability distribution of $\nu\left(\boldsymbol{R}_{i}\right)$ by numerical simulation. We analyze the logarithmic probability distribution $\mathrm{d} P / \mathrm{d}(\ln \nu)=\nu \mathrm{d} P / \mathrm{d} \nu$ treating the standard deviation of this distribution, $\sigma_{\ln }$, as a measure of the nonuniformity of the distribution. A logarithmic standard deviation bigger than one indicates that the relaxation times are distributed by one order of magnitude. We treat such distributions as nonuniform.

In Fig. 1a, an example of the logarithmic probability distribution of the relaxation rate is plotted. The spread of the relaxation rate over the different orders of magnitude is seen. The shape of the distribution is very close to the Gaussian one for any Bohr radius, but the width of the distribution, $\sigma_{\mathrm{ln}}$, strongly depends on the ratio of the Bohr radius, $r_{\mathrm{B}}$, to the mean distance between $s p$-spins, $\langle r\rangle=n^{-1 / 3}$. As it is shown in the inset of Fig. 1a for high impurity concentration, when $r_{B} n^{1 / 3}$ is bigger than 1 , the logarithmic standard deviation is very small. Thus, in this metallic range, the discussed mechanism of spin relaxation can be considered as uniform. With decreasing impurity concentration, however, in the vicinity of the metal-insulator transition, the relaxation becomes nonuniform. A further decrease 

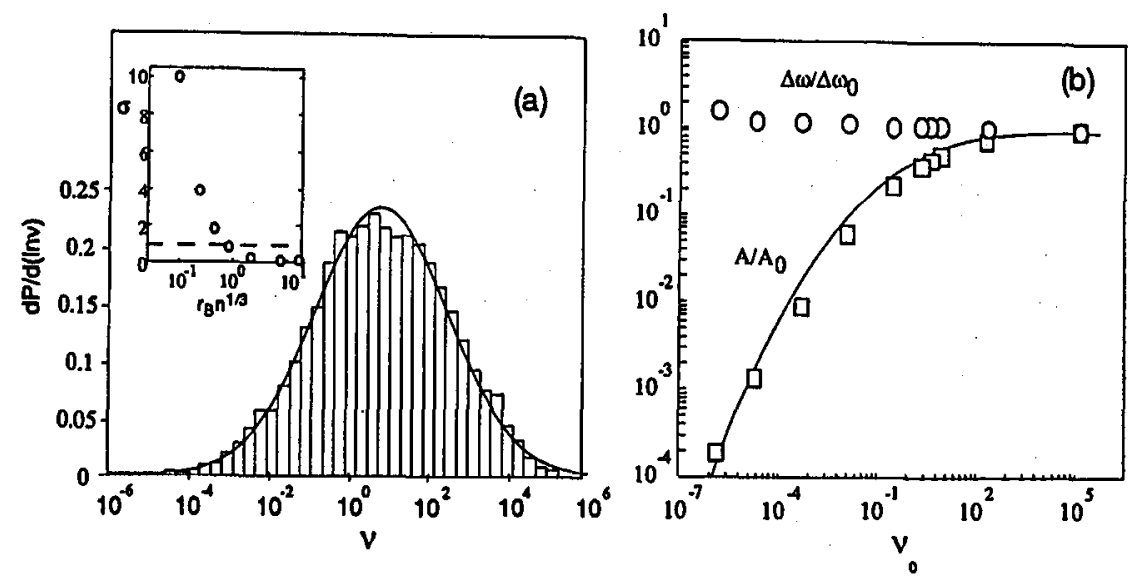

Fig. 1. (a) The logarithmic probability distribution, $\mathrm{d} P(\nu) / \mathrm{d}(\ln \nu)=\nu \mathrm{d} P(\nu) / \mathrm{d} \nu$, of the relaxation rates for the mechanism caused by the $s p-d$ coupling of the localized $d$-spins to shallow $s p$-impurities. The histogram is given for $r_{\mathrm{B}} n^{1 / 3}=0.2$. The dependence of the spread of the distribution, scaled by the logarithmic standard deviation, $\sigma_{\mathrm{ln}}$, is shown in the inset. (b) The dependence of the peak-to-peak amplitude and the linewidth for the case when a line, characterized by a native linewidth, $\Delta \omega_{0}$, is additionally broadened by a nonuniform relaxation mechanism (the parameters are the same as in (a)). The unperturbed linewidth is accompanied by a decay of the amplitude.

in the impurity concentration in the insulator phase is correlated with a sharp increase in the standard logarithmic deviation, $\sigma_{\mathrm{ln}}$. Here the logarithmic standard deviation is even more sensitive to the parameter $r_{\mathrm{B}} n^{1 / 3}$ than the mean value of the distribution. Such a sharp dependence suggests that the determination of the spin relaxation rate can be a useful tool for the investigation of the impurity concentration and the metal-insulator transition.

To analyze the influence of nonuniform relaxation on the linewidth and line amplitude we consider two approaches: (i) when the nonuniform relaxation mechanism leads to line broadening, (ii) when the longitudinal relaxation rate is much smaller than the transverse relaxation rate which determines the linewidth and the nonuniformity can be analyzed by the saturation of the amplitude only.

We assume (i) that the spectral line, characterized by a native linewidth $\Delta \omega_{0}$, is additionally broadened by a nonuniform relaxation mechanism. In Fig. $1 \mathrm{~b}$ the dependence of the peak-peak amplitude and linewidth is plotted as a function of the native linewidth. The shape of the resulting line is a sum of the contributing lines, each of them weighted by the density of the probability distribution and is assumed to be described by a Gaussian shape with the linewidth equal to $\sqrt{\nu^{2}+\Delta \omega_{0}^{2}}$. The decay of the amplitude, without noticeable broadening of the peak-to-peak linewidth, is well seen.

In Fig. 2 the dependence of the line amplitude on microwave power, for various standard deviations of the longitudinal relaxation rate, is shown. A uniform distribution is characterized by a sharp peak in the log-log plot (see the upper 


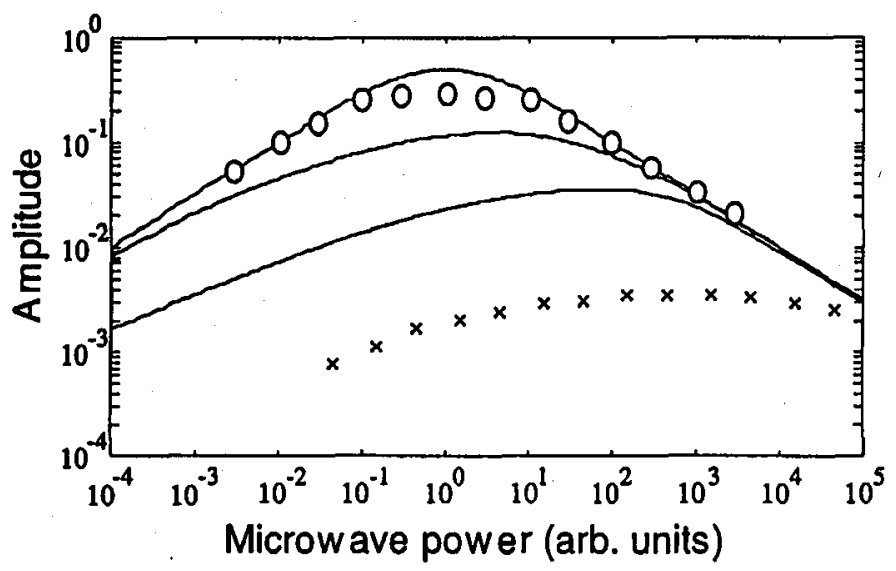

Fig. 2. The power dependence of the EPR amplitude for $r_{\mathrm{B}} n^{1 / 3}$ equal to 2 (upper curve), 0.2 (middle curve) and 0.08 (lower curve). Open circles show experimental data for the EPR signal of Mn in $p$-doped $\mathrm{ZnTe}$, crosses show the behavior of the Mn signal in $\mathrm{CdF}_{2}: \mathrm{Ga}$.

line). A nonuniform distribution causes that for a given power a fraction of spins is completely saturated while the rest contributes to the observed line. As a consequence, with an increase in the nonuniformity of the relaxation rate a broadening of the peak in the power dependence is observed.

The experimental data of the power dependence of the Mn EPR signal in $p$-doped $\mathrm{ZnTe}$ indicate a semiconducting character of the sample doping, with impurity concentration close to the metal-insulator transition. The power dependence of the EPR amplitude of Mn spectra in the semi-insulating $\mathrm{CdF}_{2}: \mathrm{Ga}$ is of a completely different type. The experimental data show here that the logarithmic standard deviation is bigger than 20 , which allows one to estimate the $r_{\mathrm{B}} n^{1 / 3}$ to be smaller than 0.01 .

The work was partially supported by the State Committee for Scientific Research (Republic of Poland) and by Fonds zur Förderung der Wissenschaftlichen Forschung in Austria.

\section{References}

[1] Z. Wilamowski, W. Jantsch, G. Hendorfer, Semicond. Sci. Technol. 5, S266 (1990).

[2] Z. Wilamowski, A. Mycielski, W. Jantsch, G. Hendorfer, Phys Rev. B 38, 3621 (1988). 